Universitäts- und Landesbibliothek Sachsen-Anhalt

Digitalisierung von Drucken des 18. Jahrhunderts

\title{
Erinnerungen an eine Freundin
}

[Christoph Martin Wieland]

Berlin, 1758

VD18 10442995

urn:nbn:de:gbv:3:1-257452 


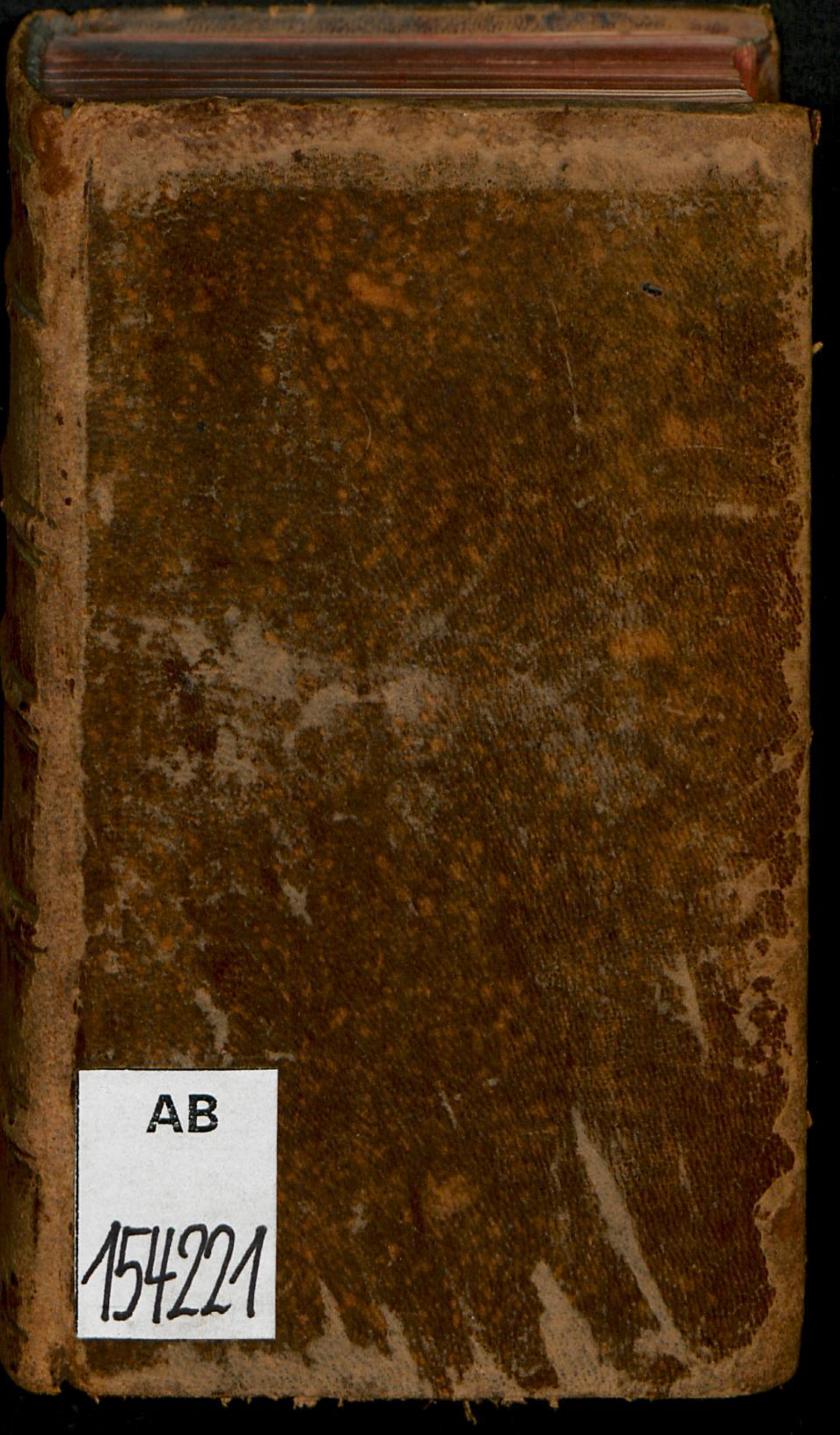




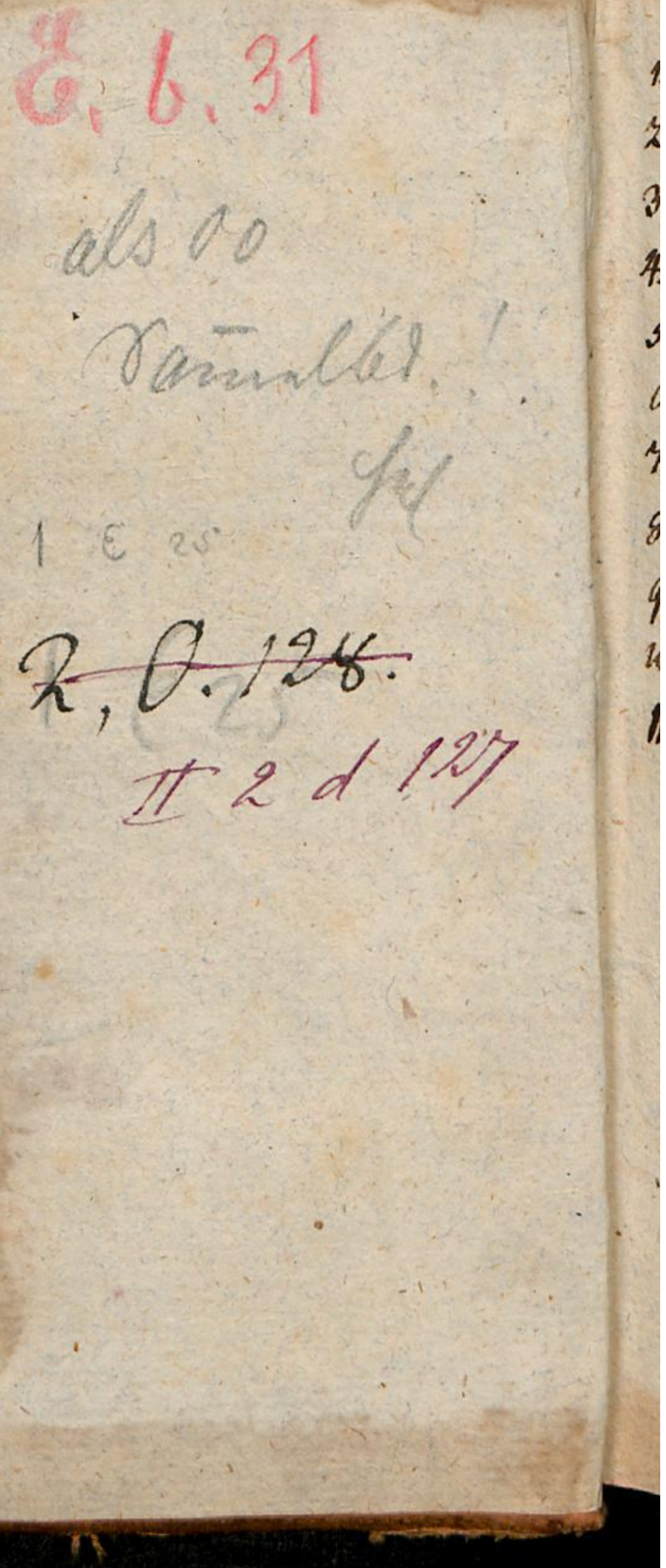




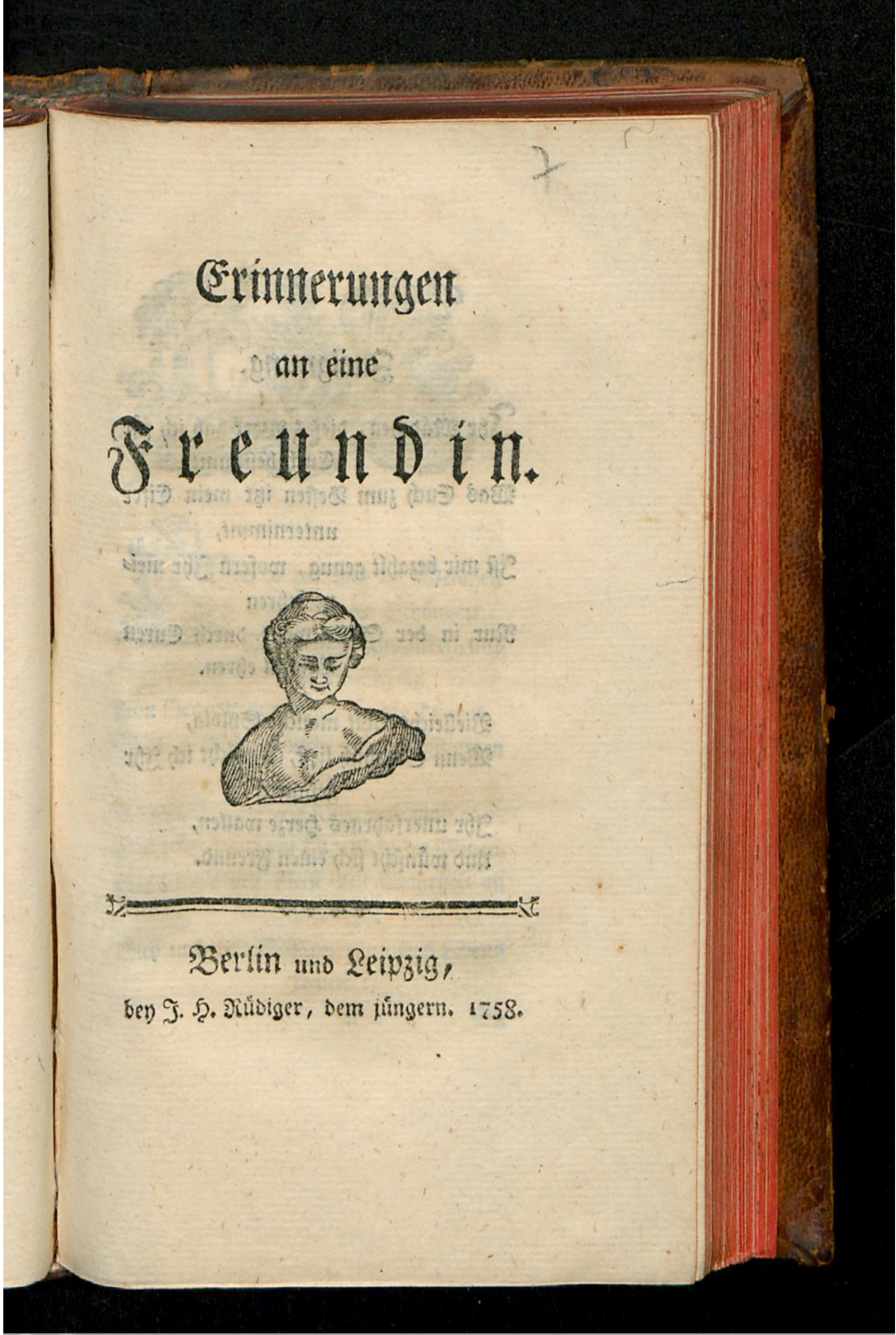




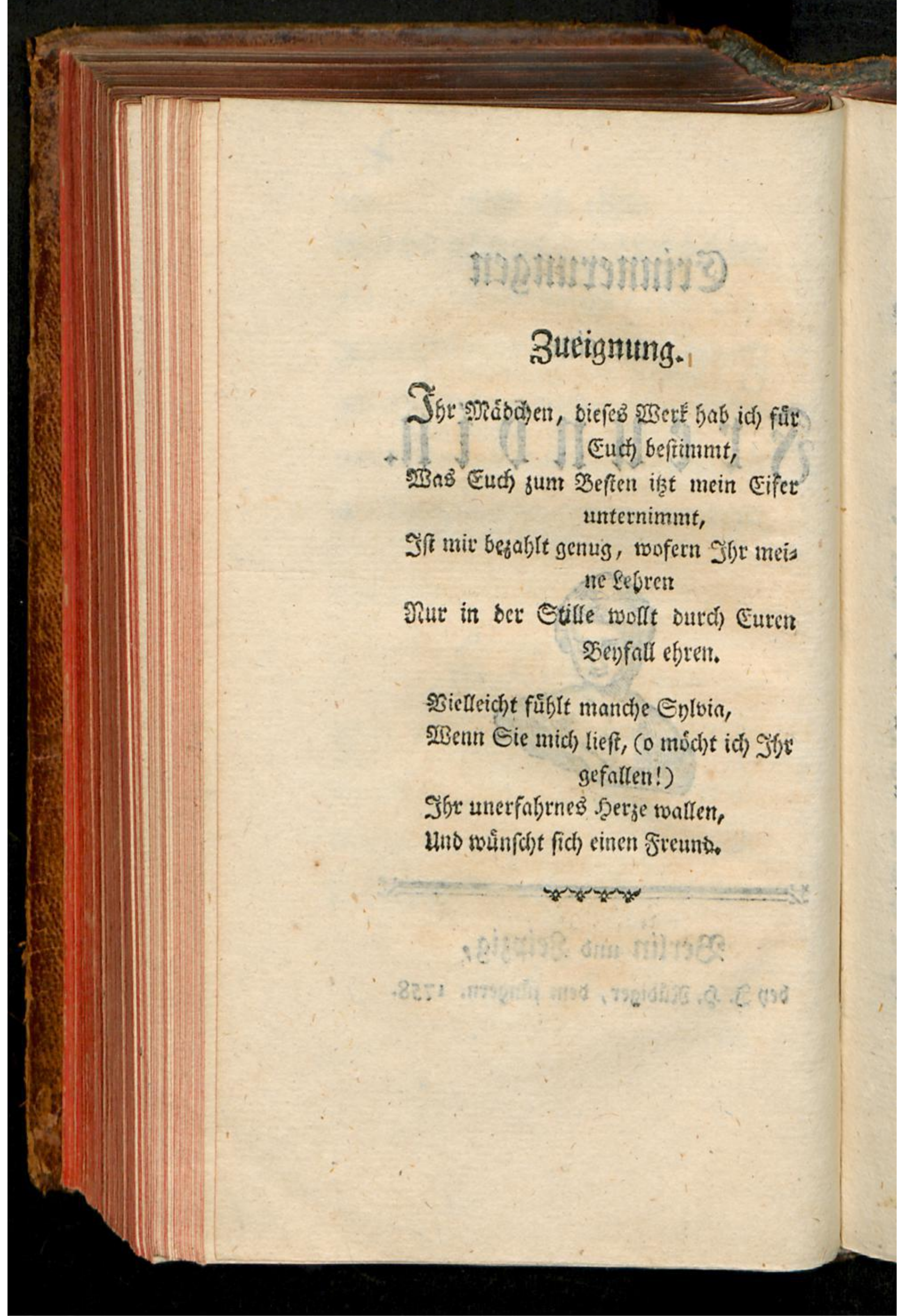




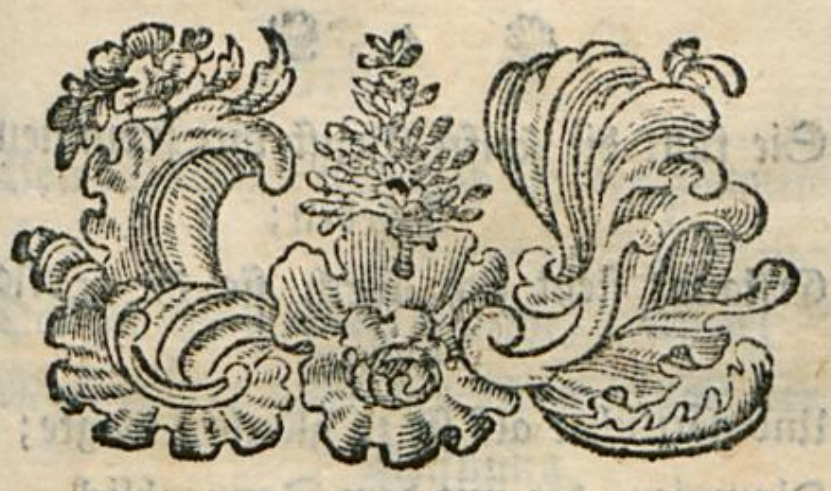

ifet

reis

ren

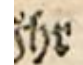

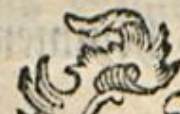

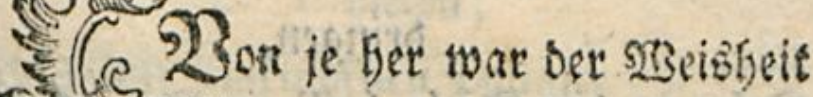

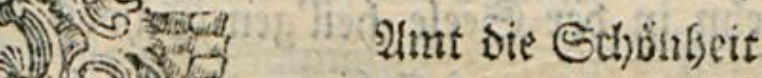

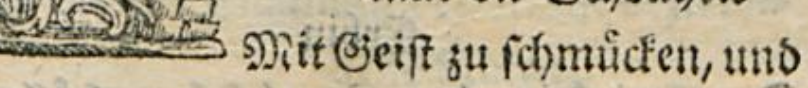
ifre ein Gefoly

2on ङiratien 3u geben, bie bie Sugens Gebabl, und bie nictet mit ben 13 antgen strelfen.

Sie mact)t bie Eecle folyon uno malyet fie Mit jeber fidytbatn It tigend in bie Dinen, Sie frrablt Den Geiff mit sisabifient an unb lefit

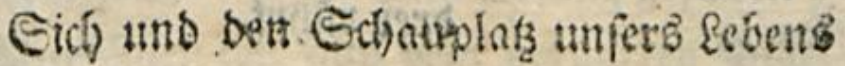
temnen!

श 2

Sis 


\section{4}

Cic fünt bie fanfte 3 ruft mit froumet

Srieben;

Exftaunt fieft fich Durdi fie bie Eeele (c)jontur

Itno gottlicher atz fie il Slauber wagte;

Dinmpier, Die mit bem Eonnenbfict

Durch biefe Fatben, bie unz bentunet, Dringen,

Eegn in ber Eele byel'gen Echooá bie Striin

Der Enigteit, Den (Engel fid) entfütlen.

Dir gab ber feimmel unter feinets Ginben

Sie luffould in ber 2 mmutly fanftem Edjmud

Die ofne Sáttlichfeit out ferent.

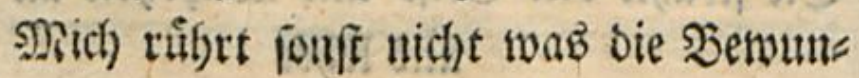

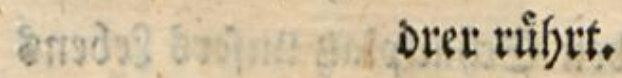




\section{(2) 5}

mbett

Eeele

jte;

E

mett,

bie

Inetr.

moS?

intets

ftem

outte

20n

Bon 23 sinflyen frey Gab ids ben golbutet Joinp,

Der um bie (jorofen raufht, gefehen;

Sich ribret niche ber fleme Stols bet. feoffinung

2th Ellaw ber Cflaweft ansert zu get bieten;

Nach) ifite marmornen \$attafite

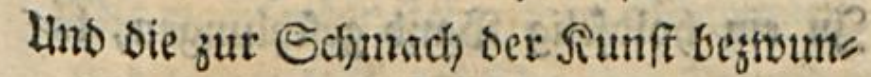
gene Natur.

2imein mich rutgrt in anmutfoseotfet? 2urgen

Die unverffellte fich betwuste Lnfchulb,

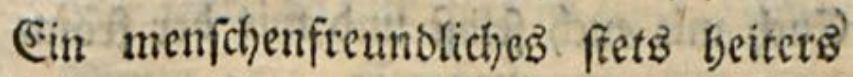
Eardyeln,

Lins auf bie reizenbern Geppielen

Ein slick, Den nicht ber Эeeis sergiftet. จur folchen mo̊ge mein ङejang gefalten

Nur ifnen Tolf aus sem getưbten 2uge

Serenens Lunglick ểe Sbtain entlocfen!

ม 3 IIno 


\section{6}

Ltus unter ifnen bit, o freunsin! Sir, Du aud) boin Schidffal mir zum \&obne Befchentte Greuntin, Deren Silb fich oft In ernften Standen, menn mid) \$ans thea

Itno Dafite und Immenens Sblict bes fuchet,

Scinjugeferft und mit ben wertben Seelent Sin ein Golofelig Sant geft)lungen, la: chelt.

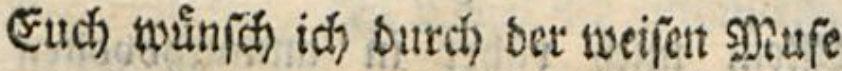
Iiraft

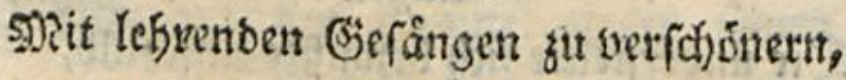
(Eud) mit euth fellof und mit ber froms men Sugens

Itno ifren freuben noct) vertraulictifer

3u madjen; eud) in veigerfintten saus ftern

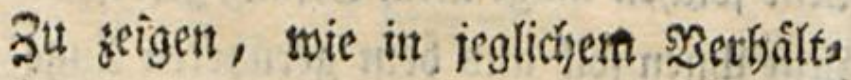
nif̧ 


\section{\% 8}

Dir,

ofne

(c) oft

Wan:

i bes

Šeelent

1, Iㄴ?

Mitupe

inetts,

froms

$\mathrm{et}$

S) XIt:

tbâlts

Die
Sie \$flidst eut mirt, uns trie enflabne

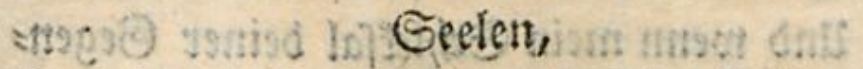
Set Sorfidft treth aud iffe Reibest

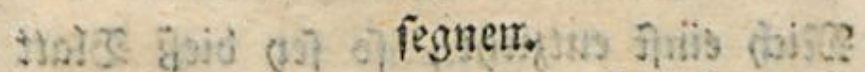

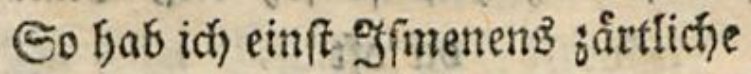
sioch unverfuchte Seele ausgebitoet; Gie übt' im milben Sonnenfot)ein ber SBabrkit

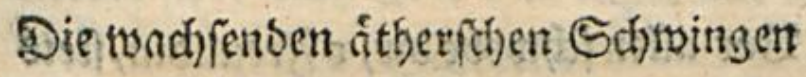
Hund lernte bald im Sha den freund ets reichen.

5. Du gleich jener liebenswertfo tins. (c) ullo,

Sen jetzt, ba uir Simene feglt, Ifmene: grimm Die Erinmungen ber treuen Fteunb/daft,

Der Freunbrhaft Die sum feften भingen mert

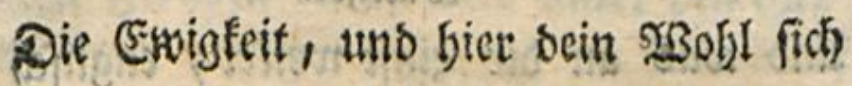
macl)t, 21 4 Int 


\section{(4) 8}

Unb bruflete fie in beine tweid)e Secle. unt wenn mein Schicffal beiner Biegent: mart

SMzich einft entsiefft, fo fen bies Slatt sir oft

Cin nicht unwertbes Denfmal meinet Freundichaft.

Zor allen fchwebe bir, o Freunsin, ffets Der Seeten Gobe şutroe vor den 2ugen, Seichau ifn oft Den beiligen Beodanfen, Dii trầgeft ber Bottheit \$ilonia, Die Sernunft,

Die bobe Straft sie $23 a b t b e i t$ fu et: fennen

Ind beike Neigungen nach) ift ź bilbert. Der Ecjimeichler higt, ber deine siofen= wangen Eltub was an bir cinft welfet, englifen nent. citis - A : is Nur 


\section{(10) 9}

Satr burd Den (Jeiff, nur burch bein etwig Efjeil

Sifit bu bem Eetaptim verwandet und

Slatt kinftig

Die flige Geppielin ifrer Frente.

einet

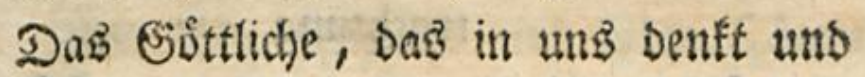

958

fets liebt,

Strebt fets nach Gott uno rubet nur im Schatten

Der Flliget feiner f̧ulb, bie \$seltett Decten.

Dft fer in einfamen geweybten Stum ben

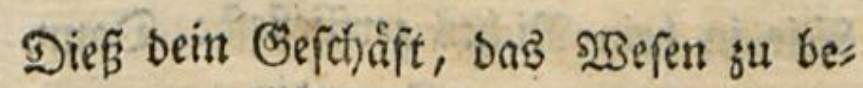
trac) ten,

Durd) weldhes bu des Tages golbues Richt

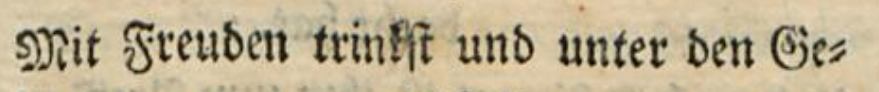

lifitis

(ch)ipfen

Dein liebengatwirbig 2ingeficht erfebft.

Deur

$$
\text { 24 } 5 \text { खं? }
$$




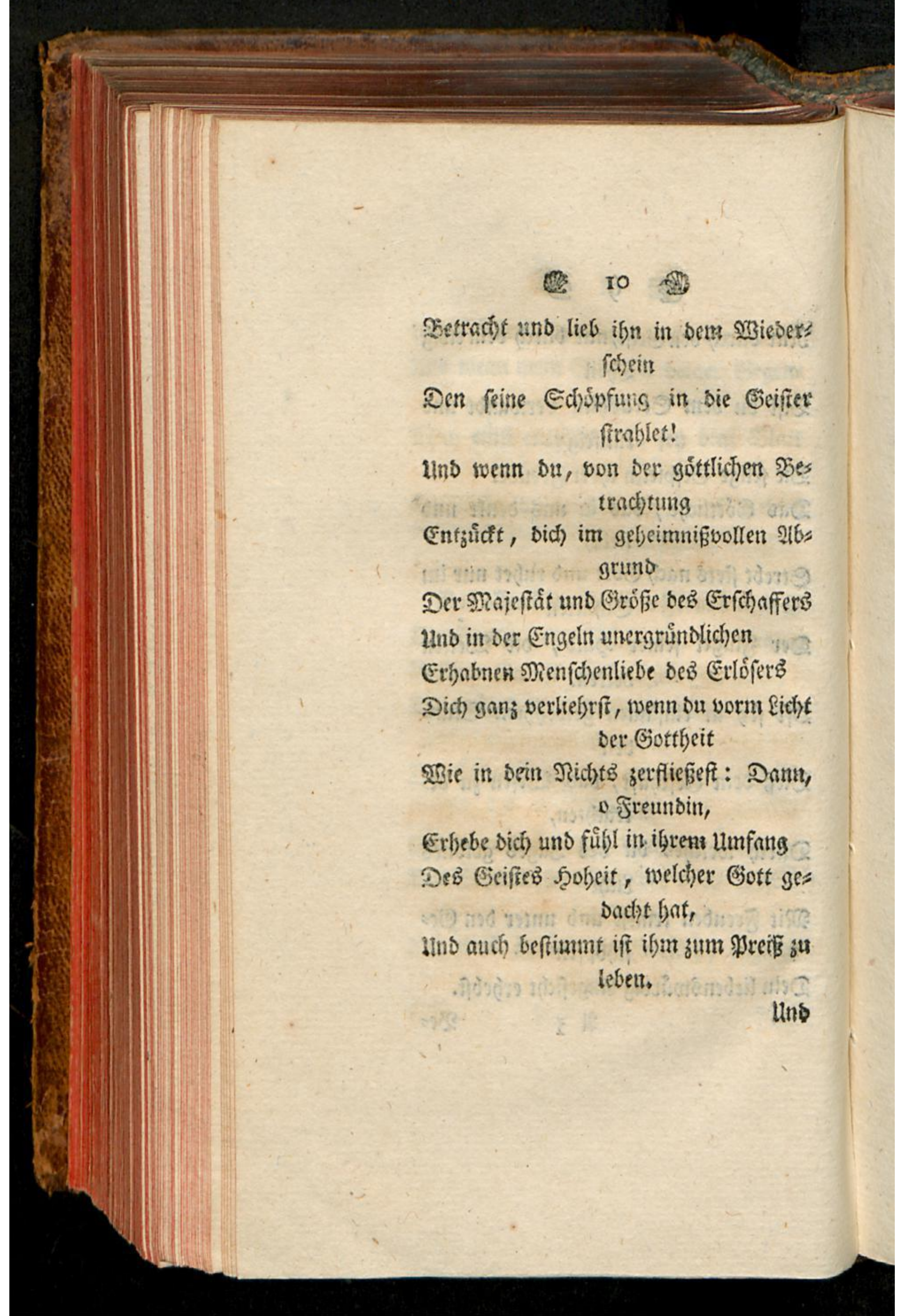




\section{II}

ebers

eiffer

Sies

2ib:

Iffers

342

เรื

Ritfyt

rami,

ng

t ges

绉

Uns
Lino wenn ou Den Giebanten, ber ben Eמ geln

Bin ganjer Scimmel iff, ben bir gebenfeft,

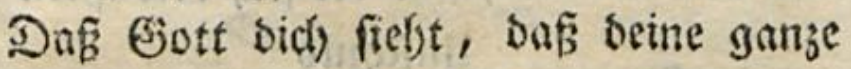

Eeele

Nor ifsm entburllt mit ifren Shaten liegt:

Co moge ftets bein unbeffecteb Jets In ftiller beiliger Entzicfung wallen! D! triemals lä́ dir biefe Seligfeit Der Eeel entmenten die DaB Dafent Cinttes

Zu jeber 3eit mit Siul) gebenfen sarf! Sie übermirget ein Ëebirg von Reiben,

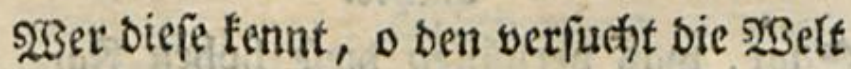
Llmjonft mit ifgren Libertündyten Freus betr.

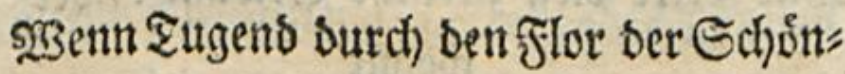
beit focheint. $23 a$ if wobl liebenstwurbiger aks Sie?

Ein 


\section{(12) 12 政}

Cin bentents 2uge, bab mit etrufer sin mutis

Uno mit ber Nojeffit ber fith berwußfent unfthuld is ?

Etiflfchtweigend tabelt oder billigt, szie miatitig ftrablet $e b$ in eble Eees len?

Sft lebit ein \$lick von einer \$antbea Genoltiger alš eineż Şingegen fief), Den ftrengeften Contraft

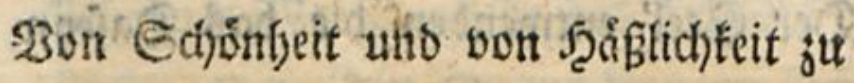
feffen,

Rarciffen at, bie gleich) bem Frübling

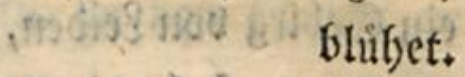

eagt, toas berfuiffen biefe goben fats bent

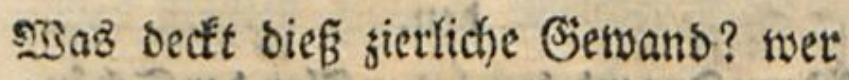
wobnt

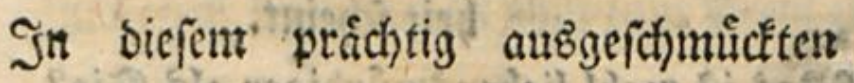
f̧aufe? = Eirt 


\section{e. 13}

Ein Sempel wont \$orpbir bect einer

Affen!

jtert

Sin ifren Itugen laurt Der Durft nady Eiegent

tho jebe Mine giebt fich felber ßenfall. Die NRißgumft fojielt bervor nus igren Sächeln

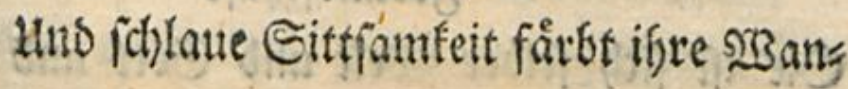
gent.

Die Gratien entfliefn, to balb fie (prid)t.

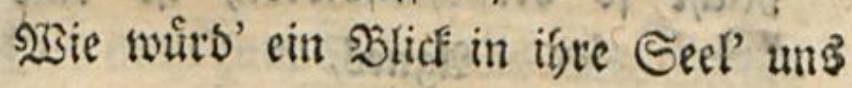
felvecten, 2isen fie, enttleibet von ben Frúglingą farben

DeB̉ (h)ơnen \&eiber, unferm Itug ets ichiene?

ver

ten

tent

Eirt Lins 


\section{(16) 14 S13}

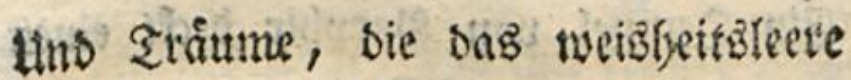
Sุirnt

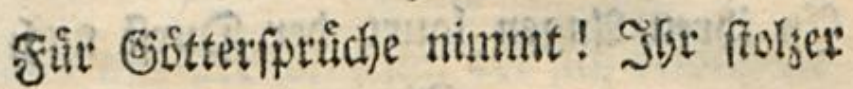
Sulen

Echwelt nur won siebe su ifye fetber auf. Scier iff fein Siaum für Sugend voer Freunif (t) aft

Eie ift fith folber șelt und biott und alfer.

Dicht fo verachtenstwerth) if eine 2tgnes,

Edjön olyne Seele, Ghăgens wie bie Siofe

2tn ifyer Sruft, beredt wie eine \$ttppe.

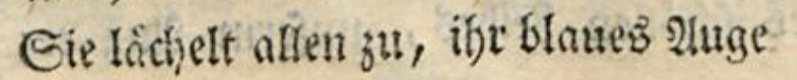
Sagt allen nichts, uno niemals wibet: (pricift

Sibr SRofenmund dem feelenlofen Suge. Eo ferht auf einem marmornen Befferfe 


\section{(15 15}

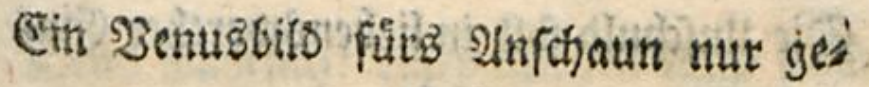

$$
\text { madst, }
$$

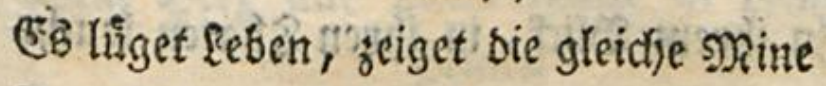

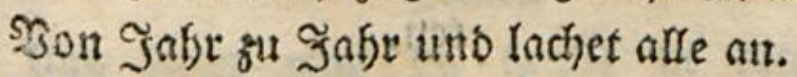
ISBie tief ernieorigt ibe etch, the S?as ciffert!

Sief unter bie Deffimmung eurer Eeete Shef unter Die Sefimmung Des: Ges fohlectits!

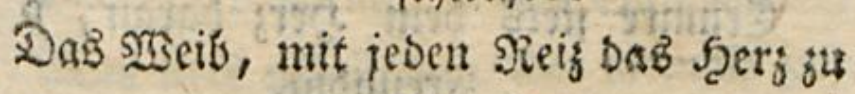
fohmerigen,

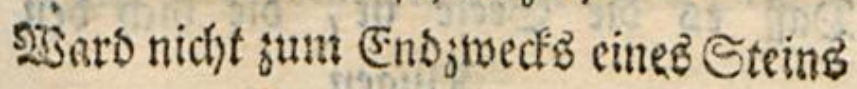
erfofaffen,

Tioch) but sem Sánbeth geiffberaubtet Silfie.

Die Edjónen fins gemacht, ber Nienfófen Situmer

Sintwegyullicheln ober gu erteidtern,

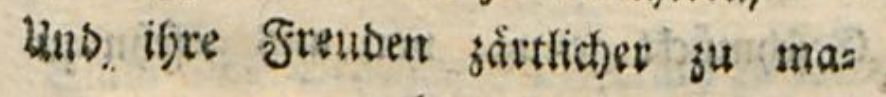
(1). chent 


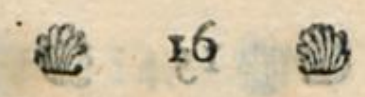

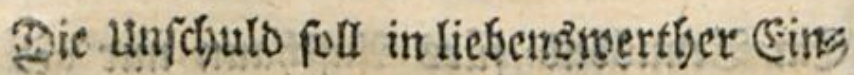
falt

In ifrem \$lide, in ifren SGaten leudty sto slin tgly telt.

Dft bat bie månnlidie ju gluifnde ₹us genl

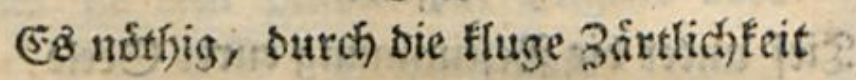
Der weiblichen barmonifcher zu twerber.

Erinnte fets bein Scers Daran, o 1. Guch Tho Freunsin,

Doñ es Die Secle ift, bie bidy ben Silugen

Beffintig macht. Die äufre Schot. bsit if:

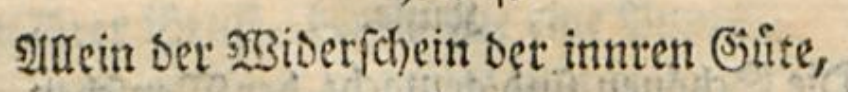
Der un bie Eeele Dunngerwebte flor.

Eenimmer meife! inmer fen bein Geif.

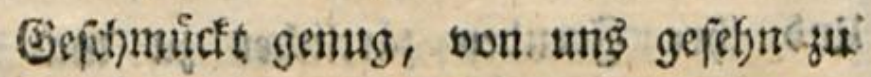
swerben,

sic

Denti 
c sins

leudity

(rios)

ใ ईน?

jecit

erdett.

in, io

b) DetR

วc) bำเ

Stite,

ot.

toieift.

thin

Denn

\section{Q 12 (iili}

Dentn wafyrlic)! cines Dein Sact' nut balbverffullt in Deinet sinen

Und fiefet was ou bentfit in beinen stugen.

Cer) unbeforgt, nwie bugefallen mógeft! Die unfduld und bie beitre Sittfantett,

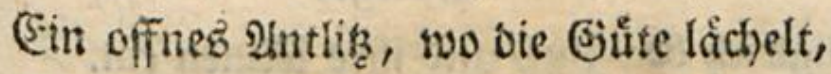
5) iuß fêt Dein Slick ein triumptitendes bemuft (e)tr.

Daß̧ but gefăllft, nie werf auf Deine 2hs muth

Dic Eitelfeit unangenebmer €chatten!

Die Echoine, bie burch Sunft gefals len will,

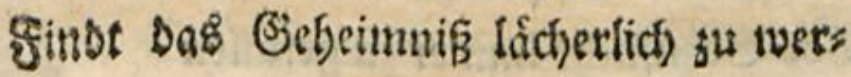
sen.

\$2 5yit 


\section{8}

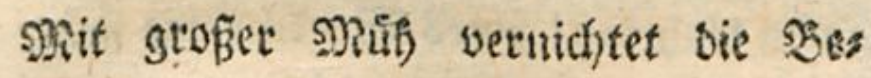
trogne.

Dab Echonfere won ify felbft und will butcl swang

Das werben was fie burch ग?atur fffon war.

Sie richtet vor bem fotmeidylerifden Epiegel

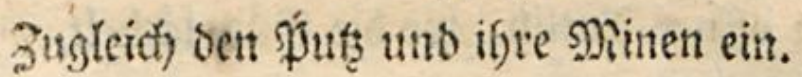

Eit jeder Slick, betwegung oder Etets lung

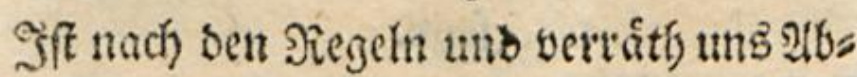
fict)t.

Eclofe iffere sounterfeit iff fteif und eh fie lächelt

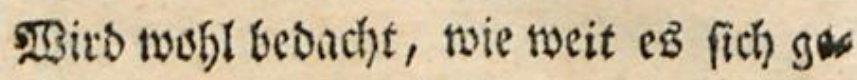
jieme

Die fprōoen Sippen zu verlängern. Bor Selinfucht iumer ju gefallen Gefält fie fich fo menig fitbft als anbert. গצsie 


\section{(1) 19}

$56 \%$

silf

$10 \mathrm{tt}$

lent

tels

$16=$

fie

30

tti.

Bie 5iție angenefm if nicht fingegan

Etella?

230 fie geft folgt ifgr bie fanfte Freube,

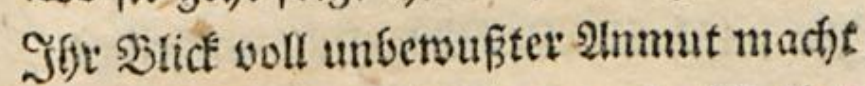
Den Fribling reijender uno Sָolfert beiter.

S) it Sittfamfeit und allgemeinet Site uno taufeno unerworbnen Rieblictfeitert Geminnt fie jeber scerz uno tweif es nicit)t.

Die fuct)te fie ben Capein bes feinets

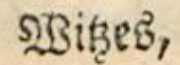

Lno mas fie fprich)t gefällt uns rifget bas Sert; ;

Dodh bơt fie lieber. Shiemalz font eit Epiegel

Der ifr voruber frano, ife freundicl) 2hige

Bur Selbferbunberung ben freunbin nen entjogen,

\$2 2

शie 


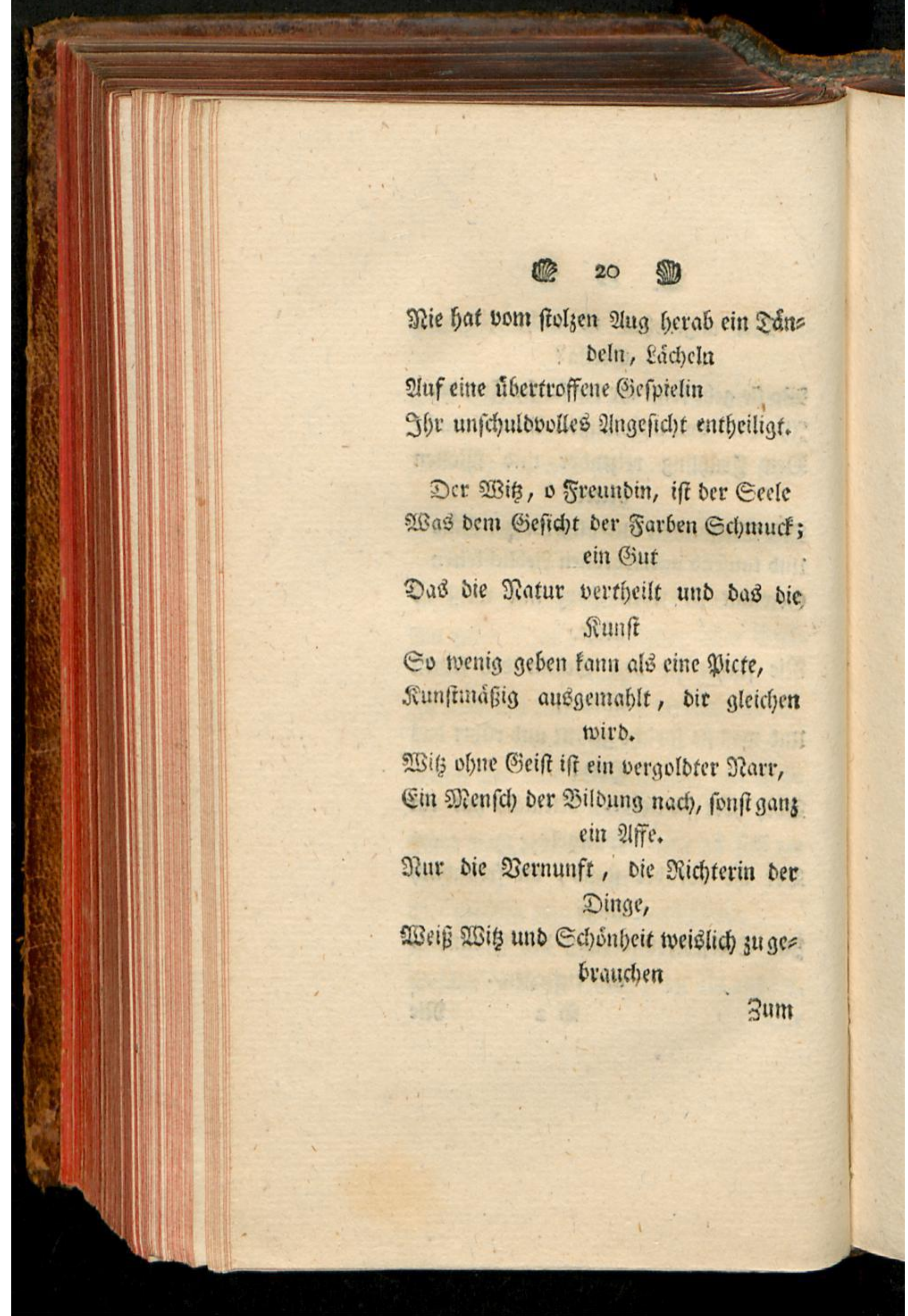




\section{(I) 21}

\&ut

If.

Ie

iff;

bie

ien

ing

jet

jes

m

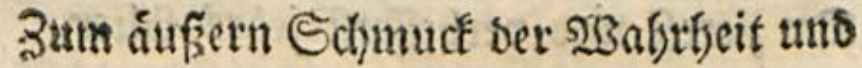
ber Gitite.

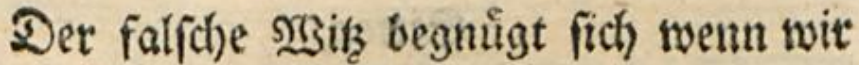
lacíen,

SBir (ad)en aud), Dod) liber ifn alfein. (Ev trill benumbert fer)n, nicht misen, llno bey nod) grofgert Shoten albె et relbit

Ejeling $e^{8}$ ifm. Denn Siluge fefn nod lieber

Die fohoine feelenlofe Agnes an,

Die immer lad)t uno toeiffe Zåbne bloft,

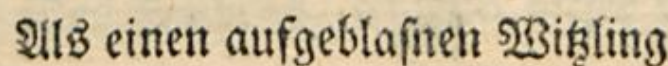

Der ewig fpricht uns niemalš benfet Şeil sir! ßernunft, bu ervig blufynte Edjontheit

Siefundseit unirer Geele, of ne tweldie

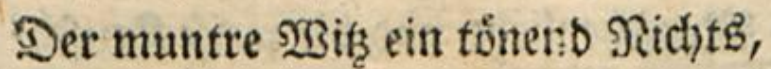

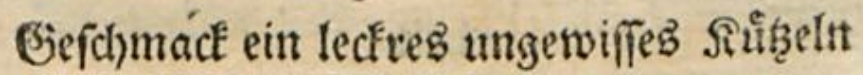
Inopgantafie ein oummer Schwasmer if.

$\$ 3$

Dits 


\section{(1) 22}

(D) jiereft und verbefferft jebes Illter, Du lefuft bie Jugeno, Wieifterin ber Sitten,

Du gię̧efe Sidyt in bie erwairmte Eeele,

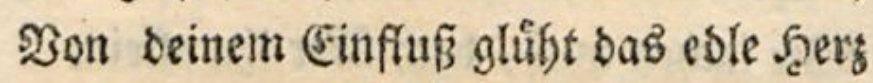
פon frommen 253 uinfhen, fiebt mit un unvertwandtem

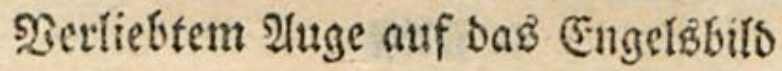
Det reinen Sugend und befretefet fich emfig \$flu gleich ju werben. Bisttliche, 2 ers numft,

sisenn bid) Die Bunde fitâtet uns etrjos bet,

Dant: fimmefi bu sie folgfanten begiets Den

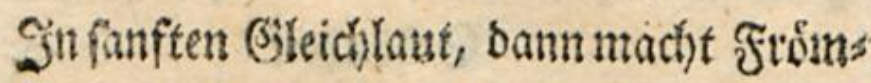
migteit

Ind Senfcherliebe und bie neife Sieu[chs beit

Die mutlige Gesulo und fanfte Demuth, \$iel 


\section{$-23$}

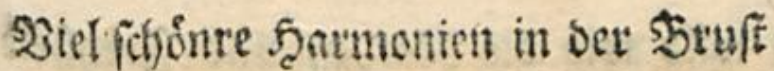

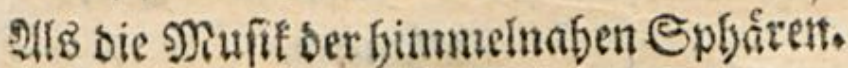

Lind wenn ber SBSis tmo bie etrabne siufe

Der S23afutbeit iffen feionen Edyleyer leis Eelt,

Damit bie blobon ungeubten 2tugen. Den feimmelglanj ber góttlict)en ertragen, Dann nimut fie jedes fecr; gefangent. (E⿱ Sun beilger Entzưctung in bie 2tuen Deß̧ Friebens, jenfeitż biefer Echaten fonne

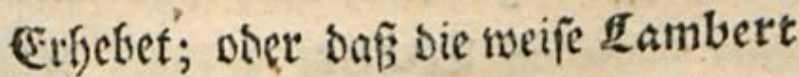
Die Sitten abelt und Gerenen bilbet; und Grafigny bie unflullo, ifree Ediwes feer, Sil nadfer uns unbeflecter Schónfyeit, reizeno

athen,

Biel

\$3 Und




\section{4}

Und witb wie bie গTatur, uns Frevgeit atfment,

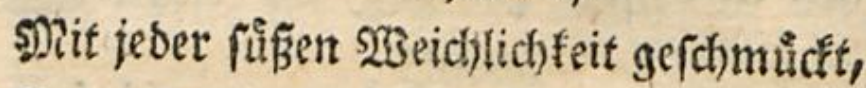
इim twilde tweißt uno כilla benemuet.

2uct) foll bir oft in Stunben ber Setrachtung

Die reiß̨entbe Beffalt Der fanften Cbamar Uno Rabels feu(che Zártlichteit ericheis nen.

Erbabne Mufter, bie ser Goltates

Der Dichter, Dir unb Deinen fahónen

Scliweftern

3um Seyfpiel gab, bamit einft eure Iodder

In eurent Sdyook von euch gul gleidjet Unifould

Bebildet merden. Retne von Debora *) Sit frommer Etille bich Den F̧igungen, Der unerforfoten Borficht unterwerfer.

Ste 


\section{(t) $25 \quad \$ 3$}

beit

ictit,

bet

Tate

jeis

tett

ite

jet

*)

in,

it.

ite
Cie twie in Gunith *) fid) bie fahoine Lins fchuld

Pitht lang wom હdhein ber Sugend fins tergangen

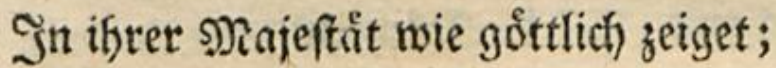

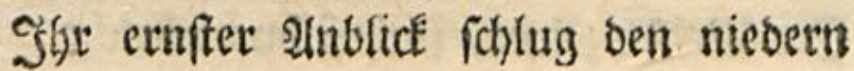
Suinter,

Die Melobie ber anmutbreidyen ভtimme Die ifn beftrafte, war in feinen Dgren Sin Donner ङottez. $=$ = So ergitterte (sin Sterblicher, wenn ibn in reinen Sưnoen Mit बㄴand umftrablt cin Engel uGerfiele. 2on biefen reijerfülten Sdjildereyen Der Tugent uno ber 23 eisheit einge: nommen,

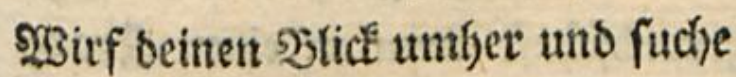
Gie bey Den syenfchen, und an wem fie glånz่̧

$$
\text { B } 5 \text { Den }
$$

*) Eiebe ben KToab und bie Gůnoflut. 


\section{(9) 26}

Den efre. SBer bie Sugend thátig preifet Der fen bein freuto. 3war Mufter wie bie STupe

Sin Dichtrifhen Der Sachtwelt Geilgets Ràchten

Cie șeilen jeiget, fucheft bu vielfeidyt Imionf ben Deinen zeitgenoffen. Nur felten maget Die SRatur Serenen und biefe fetoft find wie ber beitte Nions Pidift ofye Flecten, bod) bie Flecten glíngent.

stmein jerfitent wirft bu bie 3lige finbert Die wir in ein volftommes sillo vers fammlen,

tho ouch serfitent verbienen fie bein f̧ers,

5) ie Freunbichaft fann in cinen Sirans winben.

(E) willig, sielen bein freundicthaftlich Shers 


\section{(10.8 27}

eipet

wis

gets

it

Dtis

Fen

it

lets

ein

มก:

(ich)

3u

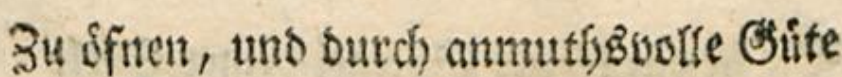

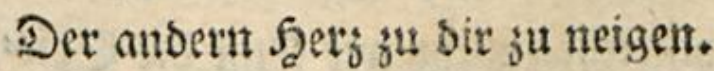
Dod) wäble bir nur biefe sur \$ertrauten,

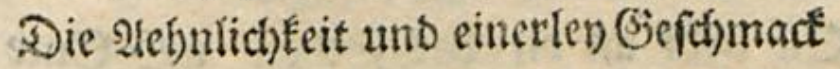
2n Dem wa Sll wie mit ongebofrner Sympatbie serbindet.

Entsuctend iff fure eine fojonte Seele

Das Brlicf Dem botben Sufen einer

Freunoin

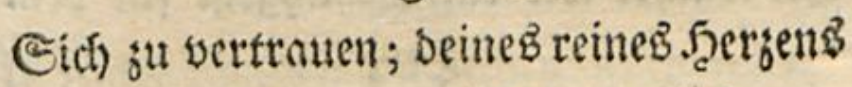
Geheimfte PReigungen ifr aufjudecten, uno beine Freuben fo wie beine Sorgen S)it ifgr su theilen! unjertrent mit ifse Den engen PFad zur Erwigkeit ju freigen. Sie lefret Dich mit SGaten mefre als tes

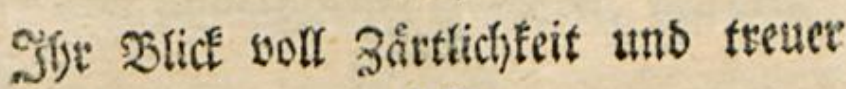
Gitite

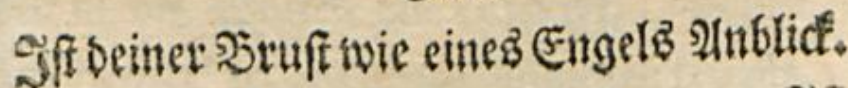
Siti 


\section{(13) $28 \quad 3$}

Sie ift ein treuter Epiegel seiner Eeele Int fohmeidhelt nicht wie fonft bie Epies get pflegett.

Eie liebt an bir baร Echoin' und Gatute nut,

Ind will siel rieber beine Fefler beffert

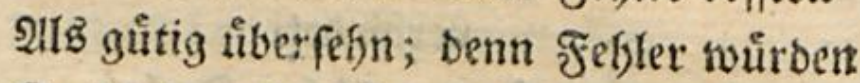
Dod) niemals f(hoำ unb wenn wit fie auch liebten.

Eie wachet wie ein Echutgeiff für bein frets

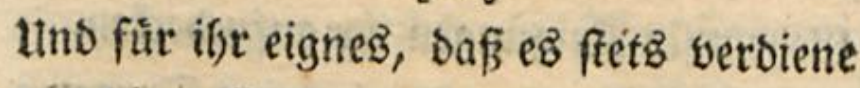
won bir gefiebt uno nachgeabmt su wers Den.

Eo war einft Ђove's unb Clarifla's Freunbftyaft,

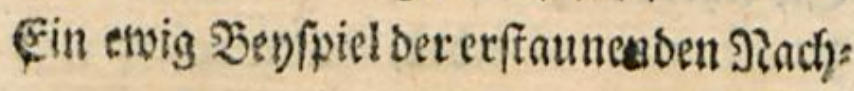
welt: 


\section{(19 29}

Sserachte fets den Edymeidjer in

Der Enrve

Ser Freundichaft doer siebe. Eeine

Stute

Reben

Eind ein bejauberns Bift, Den SOfren

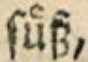

wit

rbent

Der Unichulo tobfich. STSem or bi(h)

utch

In peiner albernen sintyictung

Zum Engel macht, weกn maร bu Gutes

beint

$$
\text { baft, }
$$

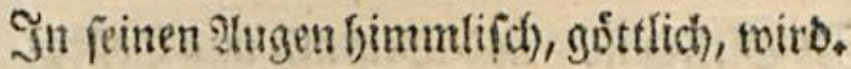

ene

Eo glaube nui, er femet beine Ed)waiche,

set:

Unb nennt, bich um bie Mienfichen ju bee

trigen,

a's

W3ie Entan Even tbat, Dich eine Biottim.

d):

Der (c)machtende \$3erefter, Den bein

Rächelin

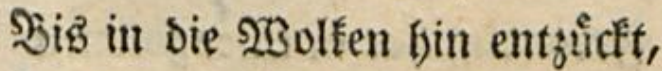

Dȩ́ fleine 


\section{(1) 30 5113}

Der Siatupe gleich bie um biesiofe fdyletdyet, Der if ein Tfor, zum wenigften eis sironter.

Ein Siluget ferme Den wafrest $233 e r t h$ Der Dinge

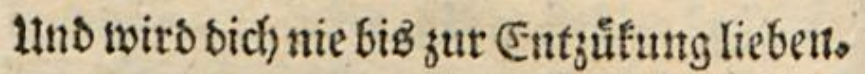
ఇicht leicht betright ifn eine Dulcinea.

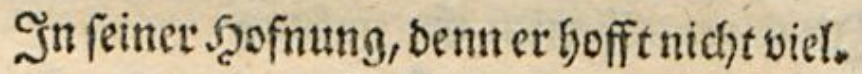

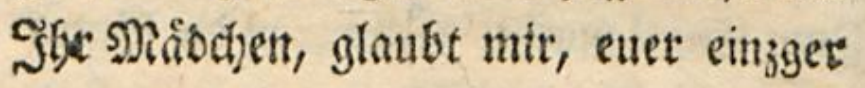
Swerth)

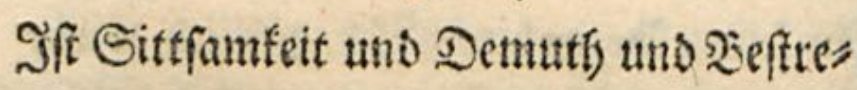
fung

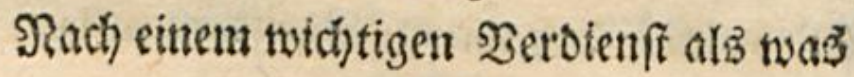
Die Atugetr

Die ఇich)t burch farben pefen blendet. und wollt ifr auch won andern als yon Diefen

Gieliebet fenn, fo liebt euch) felber minber. Dick), freunbin, bat in einer golbnet Stunde 


\section{(1) 3}

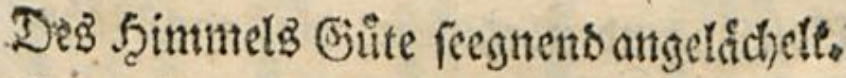
Siein Pieitz Darf Did) Der ttrgendbaften (Eiss falt

(Entjiefn, sergeblich) maditt bie blose (sis telfeit

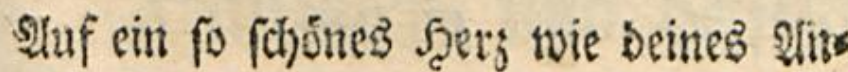
fprudy.

Du Gluffeft in ben fanften Influengen Der frommen Sugent, auf ein finftig Senfpiel!

In beinem 2fem witb einfte ein fluger Mant Eein woblgebrauchtes Reben fefferfifilen; Sadi) Dir wito fict) Dereinft an Dcinem Souren,

Der fod wott mitterlichen fretben aufs wallt,

Der fohonen Sochter weidje Eeple bifloen.

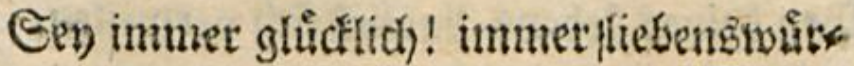
Dig!

Etetâfer beit Sers mit einet Engets̈nache গon 


\section{(1) 32}

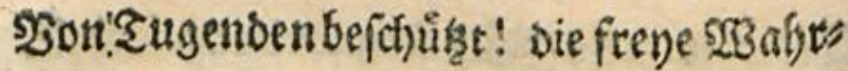

beit

Die Seufdrgeit mit Dem fittfanbeitern Inge,

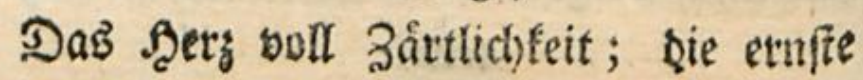
Silugheit

Die 210lerblicke in 3uk unft witft, DaB \$Ritleib und in Sicht gefleibt bie lun (c) uld,

und Sirommigfeit, Die bimmilifche (Seftalt, Die Dich) an beinen lurprotung oft erinnert: Die follen etwig einen lichten fireis Lum beine Eeele machen; fanfte アiuthe Soll bich in ifre Siofenfligel bullten; und Seraphim, Die ungefefnen zengen જon unfern Sbaten, follen bimmlipas låchelno

Sen Deines Rebenz 2nblick fich) verweilen.

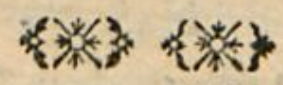




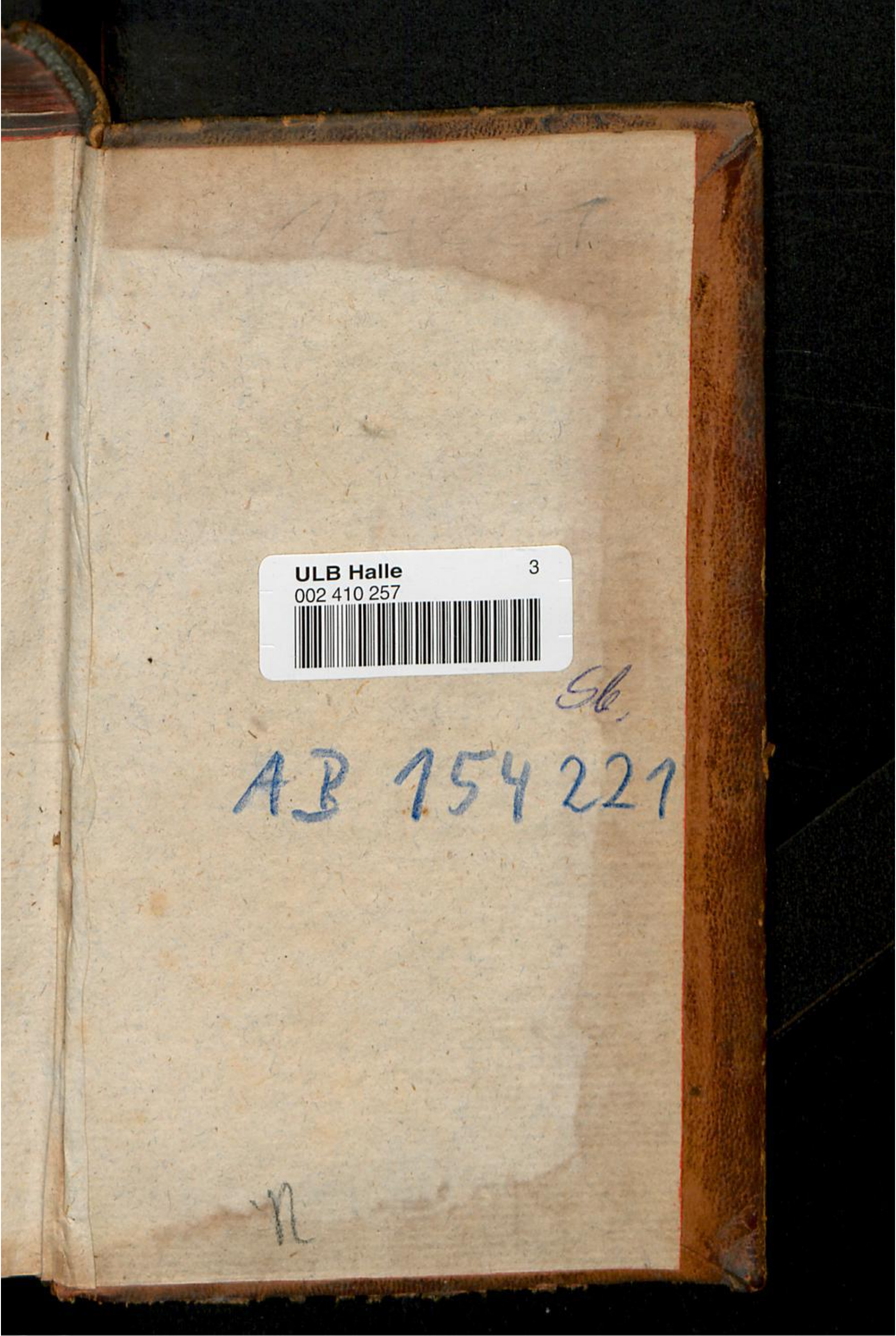


